Article

\title{
Can Climate Skeptics Be Convinced? The Effect of Nature Videos on Environmental Concern
}

\author{
Axel Franzen * and Sebastian Mader \\ Institute of Sociology, University of Bern, 3012 Bern, Switzerland; sebastian.mader@soz.unibe.ch \\ * Correspondence: franzen@soz.unibe.ch
}

Received: 3 March 2020; Accepted: 3 April 2020; Published: 8 April 2020

check for updates

\begin{abstract}
Much research has demonstrated that videos can function as primers or nudges that influence attitudes and behaviors. Studies to date suggest that this includes influence over individual pro-environmental attitudes. However, the existing evidence all stems from samples comprised of university students. In this paper, we describe the results of a randomized online experiment in a sample of 468 climate skeptics. We presented 3-min nature documentary videos that highlighted either the beauty of nature, the endangerment of nature by humans, or a mixture of both. The results suggest that the mixed stimulus video, which shows first the beauty of nature and then its endangerment by humans does indeed increase environmental concern by almost half a standard deviation. However, none of the video treatments increased donations to pro-environmental organizations. Still, the results suggest that nudging by video also works in samples of climate skeptics, which demonstrates the external validity of former findings.
\end{abstract}

Keywords: environmental skepticism; environmental concern; environmental video primers; environmental education

\section{Introduction}

Climate skepticism is still an obstacle to more rigorous environmental policy, not only in the United States but also in Europe and many other countries. Even those where the environmental concern of the population is generally high have a critical number of conservative parties and politicians who prevent the implementation of more strict environmental policies. This is particularly obvious in the United States, where environmental skepticism is widespread among Republicans [1,2]. But the problem also exists in many European countries where environmental skepticism can be found both in political programs and in the slogans of leading political figures of conservative parties. This is also the case in Switzerland. International surveys show that the general population ranks very high in terms of pro-environmental attitudes [3]. At the same time, Switzerland's largest political party, the Swiss People's Party (SVP), reached a $25.6 \%$ share of the vote in the last elections, and still downplays the importance of protecting the environment. For example, one of the leading politicians of Switzerland's conservative party wrote recently "There is no scientific evidence that humans significantly influence climate" [4], a statement that is in obvious contradiction to what most climate research says. This raises the question of whether climate skeptics and, more generally, people holding low pro-environmental attitudes can be convinced that more environmental protection is necessary.

One obvious answer to the question lies in education. Much environmental research in sociology and psychology has consistently demonstrated that education is positively linked to pro-environmental attitudes $[3,5,6]$. However, increasing education among a population is a long-term endeavor and this makes the general nature of this answer unsatisfactory. Hence, research has started, to investigate whether environmentally related attitudes can also be strengthened using much simpler and faster methods such as videos. Generally, video-based experimental studies have been conducted in various 
fields, e.g., in public health [7-12], criminology [13-15], and equality research [16]. Virtually all of these studies find positive effects of video interventions on socially desirable attitudes and behavioral intentions $[8,11-16]$ as well as on actual behavior $[9,10]$.

Recent examples of experimental environmental research stem from Zelenski et al. (2015) [17], Janpol and Dilts (2016) [18], Shreedhar and Mourato (2019) [19], and Jacobson et al. (2019) [20]. Zelenski et al. (2015) [17] conducted three in-lab randomized experiments showing students nature documentary films versus architectural videos. They found that subjects who were exposed to the nature video treatment displayed more cooperative behavior in a fishing common pool resource game. Janpol and Dilts (2016) [18] conducted a randomized in-lab experimental study among 81 college students. The treatment consisted of a 50-min film about dolphins. The control group saw a video about the construction of a bridge. The authors found that those who watched the film on dolphins associated themselves more strongly with the natural environment as compared to those who saw the bridge-building video. The former also more often donated a one-dollar voucher to a "save the dolphins fund" than did the latter (95\% versus 60\%). Shreedhar and Mourato (2019) [19] investigate the effect of biodiversity videos on charitable giving. They designed different visual material (sequences of photos which were voiced over) containing either bats or lions or both, and information on the human cause of their endangerment. The in-lab experiment was conducted with a total of 377 students (in nine experimental conditions) at the London School of Economics and Political Science. The results show that those who saw the material on lions donated somewhat more of the experimental endowment to a nature conservation fund than those who were exposed to the material on bats, suggesting that charismatic species (lions) elicit more donations. Jacobson et al. (2019) [20] conducted an online survey with 524 students from the University of Florida. All participants saw six 1-2 min videos that were either positively framed (showing the beauty of nature) or neutrally framed (one video) and two videos that were framed negatively (showing that nature and species are endangered). The videos were labeled with a letter representing a fictitious organization. After each video, students were asked whether they would be willing to spend money or time to volunteer for the respective organization associated with the video. The measures were hypothetical, since no real monetary endowments or time commitments were involved. They found that positive videos elicited more willingness to donate money as compared to the negative videos ( $\$ 15$ vs. $\$ 13$ ). Positive videos also received more willingness to volunteer time as compared to the negative ones ( $3.47 \mathrm{~h}$ vs. $2.90 \mathrm{~h}$ ).

Taken together, the existing evidence suggests that videos can increase the donations assigned to environmental organizations, specifically, that positive videos containing likeable animals or videos that are positively framed seem to increase the willingness to donate more than do neutral or negative videos. However, the existing evidence is still sparse. First, there are only a few studies so far. Second, all studies were conducted with university students, which raises questions about the external validity. Third, the studies that use a random experimental design involve only small subject numbers per treatment and only hypothetical or small monetary endowments to specific environmental organizations. Our own study supplements the existing evidence by trying to address some so-far unanswered questions. First, we conduct a randomized online experiment among the members of the largest conservative party in Switzerland for which environmental issues do not rank very highly. Second, we use a common and elaborate measure of environmental concern to investigate the effects of positive videos (1), negatively framed videos (2), and a combination of the two (3) on subjects' general environmental attitudes. Finally, we also investigate individuals' donation behavior. However, alongside some real and well-known existing organizations (e.g., Greenpeace), participants also had the chance to name an organization of their own choice. Finally, subjects in our study received an endowment of 20 Swiss Francs (about US \$20), which is still a small amount, but considerably more than the endowments of former studies.

The remainder of the article consists of three sections. Section 2 describes our theoretical expectations, the study design, the measurement instruments, and the data collection. Section 3 
presents the results that videos have on subjects' attitudes and donation behavior. Finally, the results and the limitations of our study are discussed in Section 4.

\section{Materials and Methods}

Former research suggests that videos can have an effect on individuals' donation behavior in student populations. In this study, we address the question of whether pro-environmental videos are also effective in a sample of climate skeptics. This is an important extension of the research in this area, since pro-environmentalists need no convincing, whereas those who are skeptical about the need for environmental protection and who often prevent more rigorous regulations are harder to persuade. There are several reasons why videos could have an effect, but also some reasons why they should not be expected to work or would have only a limited impact. First, videos might contain new information for viewers that induce a change in individuals' perceptions of the topic. However, environmental topics have been on the agenda in the media and in politics for some time. Therefore, it is unlikely that videos showing the beauty of nature or its endangerment would convey new information to participants. As a matter of fact, showing such videos might very well have the opposite effect due to fatigue. A further reason that videos can have an effect is because they function as a primer or a nudge [21,22]. Much psychological literature has demonstrated that primers can work. Taken together, it is hard to predict, theoretically, whether environmental videos would have an effect in a sample of conservative party members with weak pro-environmental attitudes, and in which direction they would work; hence, this is an empirical question. Given former evidence, we expect a positive effect from the video treatments, which should be stronger for the positive video treatment and weaker for the negative video.

We investigated this question via an online experiment among party members of Switzerland's largest conservative party. In the first step, we contacted subdivisions of various political parties in the canton of Bern in Switzerland and asked the parties' secretaries to forward an email to their members, which contained a link to a survey on the political attitudes of party members. All parties have several thousand members, and all agreed to forward our mail. The mail announced a study on the different political attitudes of party members and contained a link to an online questionnaire. Participation in the survey was voluntary. After completing the online questionnaire, the participants from the conservative party received an electronic voucher of 20 Swiss Francs that they could print and use in Switzerland's largest food retailer. The receipt of the voucher was announced in the introduction email for the survey. The survey for the other party members did not contain such a voucher. As a result, 468 or about $10 \%$ of the members of the conservative party did participate in the survey, but only very few took part from the other parties who did not receive an incentive. In the following analysis, we only take into consideration the sample of the conservative party, which is our primary target group.

The 468 participants were randomized into four groups. All four groups received an identical survey with about 100 questions on various political attitudes. The surveys only differed with respect to the videos the different groups received. The average completion time was $27 \mathrm{~min}$. The questionnaire started with 30 questions about general political attitudes, followed by either a 3-min positive video showing the beauty of nature, a 3-min negative video, or a 3-min positive and negative nature video. The control group saw no video. The films were compiled from YouTube videos of scenes provided by the BBC (mostly from the program "The Blue Planet"), National Geographic ("Before the Flood"), and Al Gore's film "An Inconvenient Sequel". The positive video was accompanied by positive and enthusiastic instrumental music, and subtitled with text describing the scenes: "Our blue planet emerged about four billion years ago-ice, wind, and water shaped nature during millions of years - glaciers store about $70 \%$ of the earth's freshwater reserves-and influence the climate as forests do - plants produce oxygen which is so important for humans-about $70 \%$ of the oxygen emerges from the oceans-oceans are the birthplace of life-there exist about 8.7 million different species of plants and animals-let us save our planet". 
The second group saw a 3-min video of scenes that demonstrate environmental pollution and destruction, e.g., pollution in cities, the burning of rainforests, large scale monoculture farming, and the accumulation of plastic garbage in the ocean. The negative video was accompanied by calm and sad instrumental music, and subtitled with the following text: "Our blue planet emerged about four billion years ago - but the environment is in danger-by air pollution — by the exploitation of non-renewable resources - by deforestation - by intensive agriculture - by too many $\mathrm{CO}_{2}$ emissions - having disastrous consequences-millions of tons of plastic end up in the oceans-the ice caps melt and seawater level is rising - floods are increasing — with the rise of temperature weather extremes increase-let us save our planet". The third group saw a combination of both, first the positive video and second the negative video, lasting 1.5 min each. Finally, the fourth group saw no video and functioned as the control group. The videos were followed by two questions about them, by items that measure environmental concern, and by further constructs that are related to environmental concern.

Figure 1 displays the distribution into the four experimental groups. Overall, 468 members of the conservative party participated in the survey. However, we excluded 79 respondents because they answered that they belong to a different party than the one the emails were sent to. This could be due to different reasons. Either some respondents obscured their party membership or the survey was answered by other respondents in the household. The questionnaire also contained a test question, asking respondents to leave this question unanswered. Overall, 28 participants did give an answer to this question, either from ignoring the instruction or because they did not read the question carefully. These respondents were also excluded from further analysis, as was one participant who took an extremely long time to answer the survey. Excluding these cases left us with 360 valid cases. However, the results were not essentially affected by these exclusions.

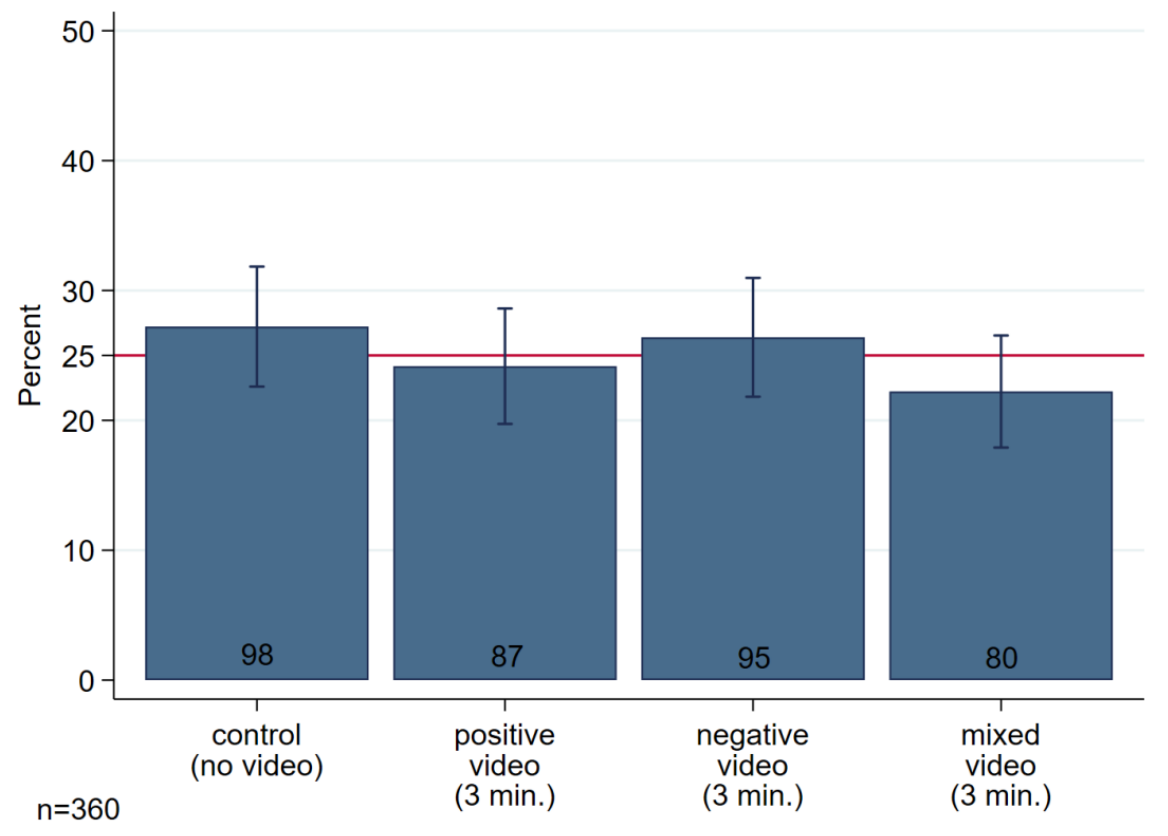

Figure 1. The results of the random assignments to the four experimental groups. Percent of cases by experimental group including $95 \%$ confidence intervals. The numbers in the bars indicate the numbers of cases in each group. The red horizontal line indicates the proportion expected by random assignment.

Figure 1 demonstrates that the randomization worked well and left us with a roughly equal number of cases in each condition (the differences are statistically not significant). Furthermore, Table 1 displays further randomization checks. In particular, we compared the age, sex, and income distributions between groups, as well as the distributions of some key political attitudes. To summarize, the sample contained mostly men $(81 \%)$, with an average age of 57 . All respondents were asked to self-rate themselves on a left-right continuum with an 11-point answering scale ranging from 
0 (very left) to 10 (very right). The average in our sample was, as expected, rather high, with a mean value of 8.04. A similar result is obtained if the answer was slightly reformulated (conservative versus liberal). Here, the mean value was 4.42. These results suggest that we were indeed dealing with a very conservative sample, as targeted. Moreover, the randomization worked well with respect to these political attitudes since the differences are very small and do not differ statistically significantly between groups.

Table 1. Randomization checks of the different control and treatment groups.

\begin{tabular}{|c|c|c|c|c|c|c|}
\hline & $\begin{array}{c}\text { Sex: } \\
\text { Female }\end{array}$ & Age & $\begin{array}{l}\text { Equivalence } \\
\text { Income } \\
\left(1^{\prime} 000 \mathrm{CHF}\right)\end{array}$ & $\begin{array}{l}\text { Conservative } \\
\text { (0)-Liberal (10) }\end{array}$ & Left (0)-Right (10) & $\mathbf{n}$ \\
\hline Control group & 0.20 & 55.7 & 5.83 & 4.30 & 7.99 & 98 \\
\hline Positive video group & 0.22 & 58.8 & 6.52 & 4.23 & 8.03 & 87 \\
\hline Negative video group & 0.22 & 58.8 & 6.33 & 4.59 & 8.01 & 95 \\
\hline Mixed video group & 0.11 & 56.3 & 6.05 & 4.59 & 8.14 & 80 \\
\hline Differences (T-test) & n.s. & n.s. & n.s. & n.s. & n.s. & n.s. \\
\hline Total & 0.19 & 57.4 & 6.19 & 4.42 & 8.04 & 360 \\
\hline
\end{tabular}

After a few questions on other political issues, respondents in the three treatment groups watched the 3-min nature videos. The videos had no voice, but came with music. In the case of the positive video, the music was very enthusiastic and optimistic, and was very slow and sad in the negative treatment. The same music was also used in the mixed video. Furthermore, the videos were accompanied by written text describing the pictures, and ended with the words "let us save our planet". All texts had exactly the same length of 74 words. The video was followed by a few questions about it and by the measurement of environmental concern. Environmental concern is usually defined as the extent of an individual's insight that humans endanger the natural environment, combined with their willingness to protect nature. This definition consists of two components, the cognitive component of having the rational insight, and the conative component of being willing to do something about it. We used a measure suggested by Franzen and Vogl (2013) [3] that consists of nine items and reflects these two components (see Table 2). These items are also included in the International Social Survey Programme (ISSP). All items can be answered on a five-point answering scale varying from (1) "not at all willing" to (5) "very willing" (for the first three items), and from (1) "do not agree at all" to (5) "agree strongly" for items 4 through 9 . The sum index ranges from 9 to 45 . The quality of the measurement with respect to its test-retest reliability and its congruent, discriminant, and external validities has been rigorously tested using a multi-trait multi-method design in another study [23]. The test results show that the scale has a high reliability and high external validity, and outperforms single item measures of environmental concern. Also, the test results in this sample show that the scale has a high internal consistency (Cronbach's alpha $=0.78)$. A principle component analysis $(\mathrm{PCA})$ extracts two components, which are termed "conative" (items 1 through 4) and "cognitive/affective" (items 5 through 9) which confirms -former study results. 
Table 2. The measurement of environmental concern (ISSP9).

\begin{tabular}{lll}
\hline (1) How willing would you be to pay much higher prices in order to protect the environment? & 0.80 \\
(2) & How willing would you be to pay much higher taxes in order to protect the environment? & 0.75 \\
(3) & How willing would you be to accept cuts in your standard of living in order to protect the environment? & 0.74 \\
(4) & I do what is right for the environment, even when it costs more money or takes more time. & 0.77 \\
(5) & Modern science will solve our environmental problems with little change to our way of living. & 0.71 \\
(6) & In order to protect the environment, the country needs economic growth. & 0.68 \\
(7) & It is just too difficult for someone like me to do much about the environment. & 0.38 \\
(8) & We worry too much about the future of the environment and not enough about prices and jobs. & 0.63 \\
(9) & People worry too much about human progress harming the environment. & 0.69 \\
\hline
\end{tabular}

Note: $\mathrm{n}=360$, Cronbach's $\alpha=0.78$, mean (ISSP9) $=27.03, \mathrm{sd}=5.87, \min .=11, \max .=44$. Each item contains five answer categories ranging from $1=$ "very unwilling" to $5=$ "very willing" for items $1-3,1=$ "disagree strongly" to $5=$ "agree strongly" for item 4 , and $1=$ "agree strongly" to $5=$ "disagree strongly" for items $5-9$. Items 1 through 4 denote the conative component. Items 5 through 9 denote the cognitive/affective component. Numbers indicate factor loadings after varimax-rotated principal component analysis (PCA).

\section{Results}

Figure 2 depicts the result of the experiment with respect to changes in environmental attitudes. As expected, the average environmental score is lowest in the control group (25.9), which did not see any video. The largest value can be observed for the mixed video treatment, in which we measure a score of 28.4. This difference is statistically significant at the $1 \%$ level $(t=2.98, p=0.003$, two-sided $t$-test). The result remains highly statistically significant after correcting for multiple tests according to the Holm-Bonferroni method [24]. In terms of standard deviations, the participants of the mixed video treatment show 0.44 standard deviations more environmental concern as compared to the control group. The difference is not big but is visible. Additionally, the positive video seems to have a small effect of 0.28 standard deviations. However, this difference is statistically only significant at the $10 \%$ level. By comparison, the values for the negative video do not differ statistically from the values measured in the control group.

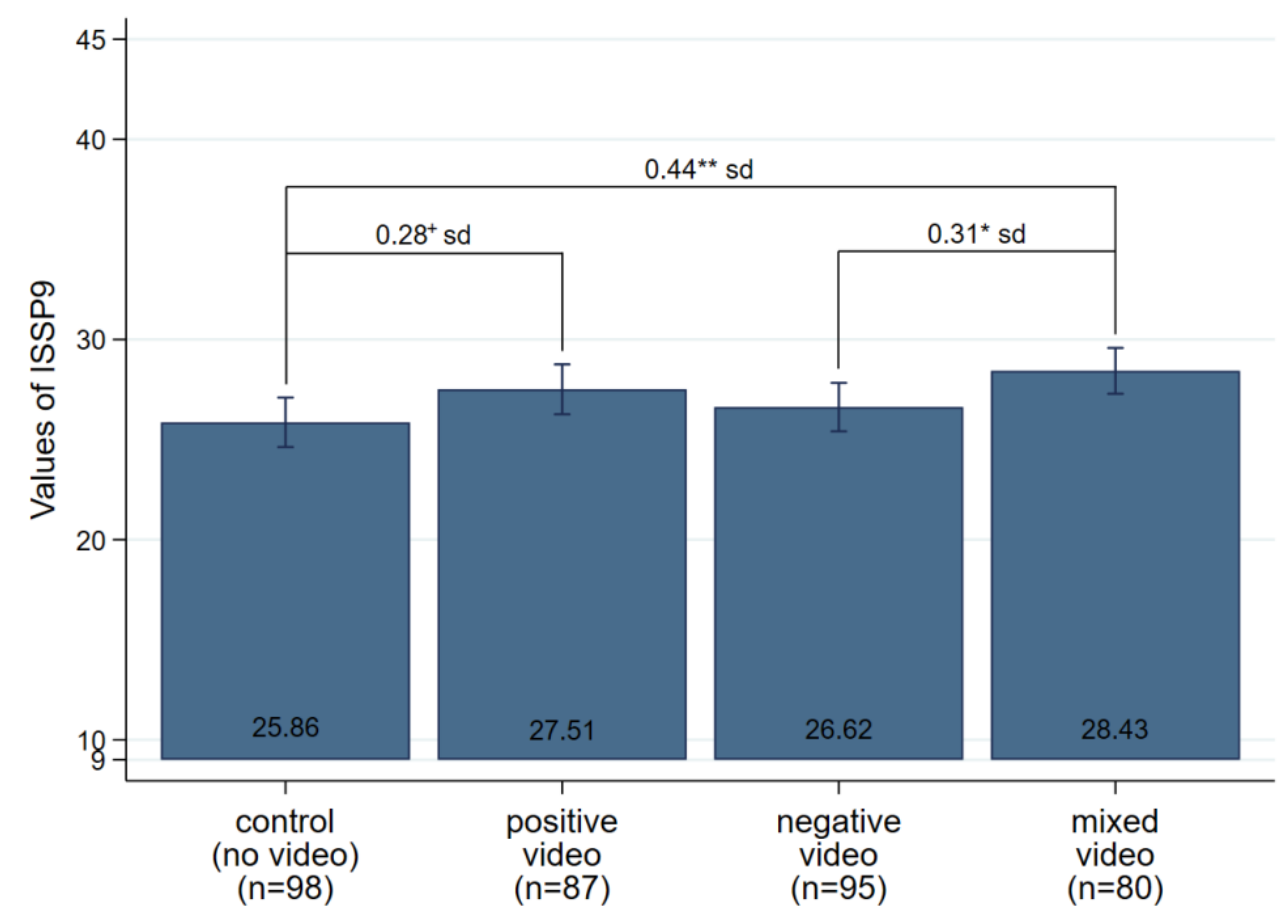

Figure 2. The results of the video treatment on environmental concern. The mean values of environmental concern (ISSP9) by experimental group including $95 \%$ confidence intervals. The numbers in the bars indicate the mean in each group. The numbers on top of the bars stand for the differences in means between the respective groups in standard deviations (sd). Two-sided $t$-tests: $+=p<0.10,{ }^{*}=p<0.05,{ }^{* *}=p<0.01$. 
The results are unchanged if we conduct multiple OLS regression analysis, controlling for various other variables. First, model 1 in Figure 3 mirrors the result when we compare the environmental concern score between the treatment groups. The group that saw the mixed video has an environmental concern score elevated by 0.44 standard deviations. Next, model 2 takes some sociodemographic information into account such as sex, age, education, and household equivalence income. Besides sex, none of these control variables has a statistically significant influence on environmental concern.

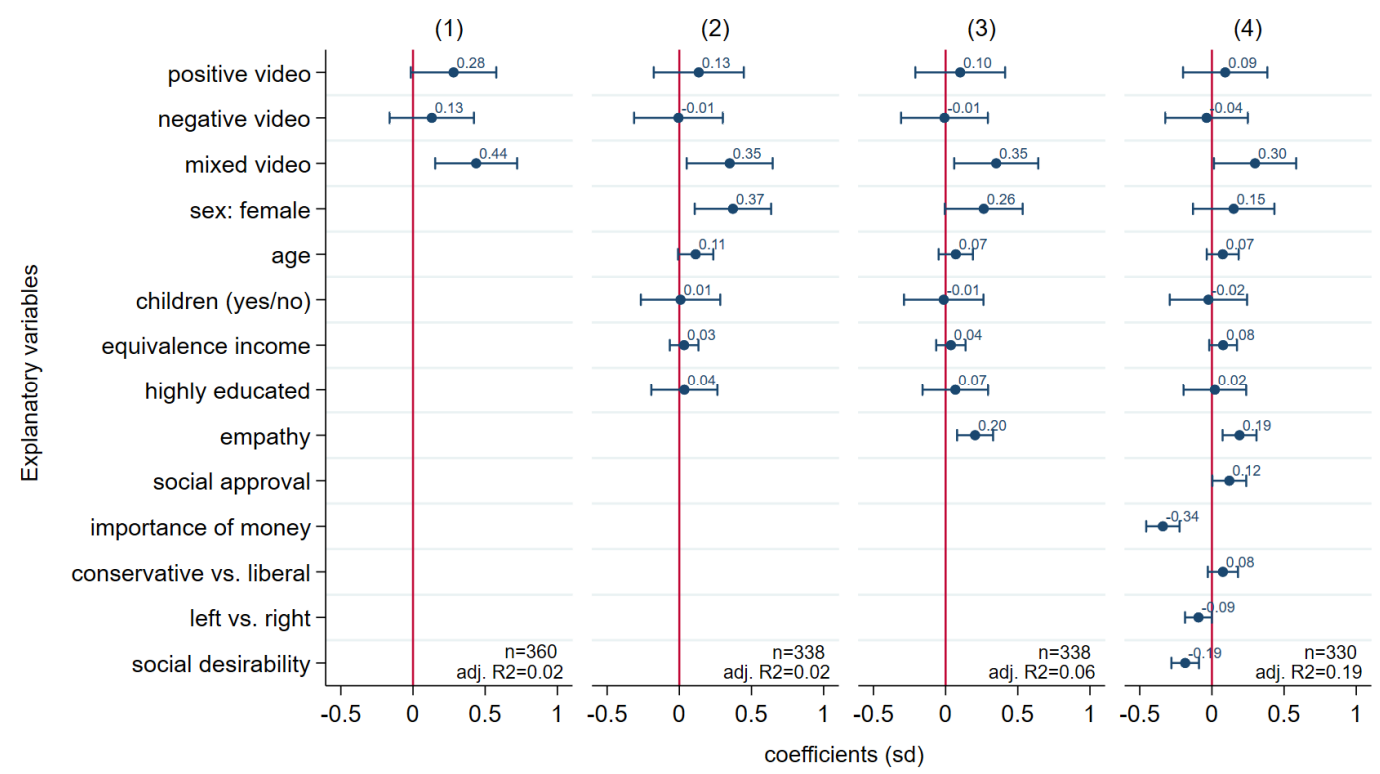

Figure 3. The results of the regression of environmental concern on various other variables. Coefficient plots of the linear ordinary least squares regressions of environmental concern (ISSP9) on various other variables. Depicted are coefficients in standard deviations (the outcome and all metric independent variables are z-standardized) with $95 \%$ confidence intervals of robust standard errors. See Table A1 in the Appendix A information for more details.

Next, we supplemented the study with the measurement of some further constructs that are presumably associated with environmental concern such as empathy, the need for social approval, and the importance of money. We measured empathy by 12 items suggested by Davis (1983) [25]. This scale (see Table 3) has high internal (Cronbach's alpha $=0.80$ ), as well as test-retest, reliability [26]. As can be seen from the results (see Figure 3), empathy is positively related to environmental concern. Furthermore, the finding that females have higher concern disappears if we control for empathy (see model 3), suggesting that the sex effect is explained by the higher empathy levels of women.

Table 3. The short version of the Interpersonal Reactivity Index (IRI).

\begin{tabular}{|c|c|c|c|}
\hline \multirow{4}{*}{ 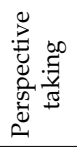 } & $(1)$ & I try to look at everybody's side of a disagreement before I make a decision. & 0.71 \\
\hline & $(2)$ & I believe that there are two sides to every question and try to look at them both. & 0.64 \\
\hline & (3) & When I am upset with someone, I usually try to put myself in his shoes for a while. & 0.69 \\
\hline & $(4)$ & Before criticizing somebody, I try to imagine how I would feel if I were in their place. & 0.78 \\
\hline \multirow{4}{*}{ 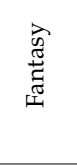 } & (5) & I really get involved with the feelings of the characters in a novel. & 0.65 \\
\hline & (6) & After seeing a play or movie, I have felt as though I were one of the characters. & 0.80 \\
\hline & (7) & When I watch a good movie, I can very easily put myself in the place of a leading character. & 0.81 \\
\hline & $(8)$ & $\begin{array}{l}\text { When I am reading an interesting story or novel, I imagine how I would feel if the events } \\
\text { in the story were happening to me. }\end{array}$ & 0.70 \\
\hline \multirow{4}{*}{ 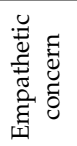 } & (9) & I often have tender, concerned feelings for people less fortunate than me. & 0.75 \\
\hline & $(10)$ & When I see someone being taken advantage of, I feel kind of protective towards them. & 0.75 \\
\hline & (11) & I am often quite touched by things that I see happen. & 0.64 \\
\hline & $(12)$ & I would describe myself as a rather soft-hearted person. & 0.69 \\
\hline
\end{tabular}

Note: $\mathrm{n}=360$, Cronbach's $\alpha=0.80$, mean $(\mathrm{IRI})=43.72, \mathrm{sd}=6.09, \mathrm{~min} .=16$, max. $=60$. Each item contains five answer categories ranging from $1=$ "disagree strongly" to $5=$ "agree strongly". Numbers indicate factor loadings after varimax-rotated principal component analysis. 
The need for social approval is measured by the eight items displayed in Table 4. The index consists of two factors which we tagged "public approval" and "private approval", and also displays a satisfactory internal consistency of Cronbach's alpha of 0.67 . As is shown by the results (see Figure 3), respondents with a high need for social approval by colleagues and friends also show higher levels of environmental concern.

Table 4. The Need for Social Approval Scale.

\begin{tabular}{llll}
\hline & $(1)$ & It is important for me that my colleagues value my work. & 0.56 \\
& $(2)$ & It is important for me that I get positive feedback, when I have done something well. & 0.67 \\
& $(3)$ & It is important for me to succeed in life. & 0.68 \\
& $(4)$ & I expect proper reward for my dedication. & 0.75 \\
\hline & $(5)$ & I do not care about how others think of me. & 0.72 \\
& $(6)$ & It matters a lot for me that my friends speak in high terms of me. & 0.55 \\
& $(7)$ & For me it is all the same, when others do not accept me the way I am. & 0.76 \\
\hline
\end{tabular}

Note: $\mathrm{n}=356$, Cronbach's $\alpha=0.67$, mean $=27.08, \mathrm{sd}=4.34, \mathrm{~min} .=12, \mathrm{max} .=38$. Each item contains five answer categories ranging from $1=$ "disagree strongly" to $5=$ "agree strongly" for items 1-4 and 6, and $1=$ "agree strongly" to $5=$ "disagree strongly" for items 5, 7, and 8. Numbers indicate factor loadings after varimax-rotated principal component analysis.

Next, the questionnaire also contained a scale intended to measure the importance of money (see Table 5). This eight-item scale is one-dimensional and has high internal consistency as indicated by a Cronbach's alpha of 0.81 . The results show that people for whom money and material things are important show less environmental concern as compared to respondents for whom money is less important.

Table 5. The Importance of Money Scale.

\begin{tabular}{lll}
\hline (1) & Money is important for me. & 0.77 \\
(2) & Money makes me happy. & 0.77 \\
(3) & I enjoy material things. & 0.71 \\
(4) & To make more money, I would work more immediately. & 0.52 \\
(5) & If in doubt, I prefer to have more than less money. & 0.55 \\
(6) & Financial security is important for my wellbeing. & 0.72 \\
(7) & Material wealth is important for me. & 0.75 \\
(8) & One can only have a decent life with a lot of money. & 0.50 \\
\hline
\end{tabular}

Note: $\mathrm{n}=359$, Cronbach's $\alpha=0.81$, mean $=26.98, \mathrm{sd}=5.00, \min .=10, \max .=39$. Each item contains five answer categories ranging from $1=$ "disagree strongly" to $5=$ "agree strongly". Numbers indicate factor loadings after varimax-rotated principal component analysis.

Furthermore, since an increase in environmental concern after the video treatment might have been due to social desirability, we incorporated a measurement of social desirability according to 10 items suggested by Crowne and Marlowe (1960) [27-29]. These ten items are displayed in Table 6. In our sample of climate skeptics, the additive index is negatively associated with environmental concern (see Figure 3), suggesting that in this sample, it is socially desirable to show less environmental concern.

Taking social-demographic information (sex, age, and education) or the measures of social desirability, empathy, the need for social approval, and the importance of money into consideration does not change our fundamental finding that the mixed video did increase respondents' environmental concern (see Figure 3).

Finally, we turn to the question of whether the video treatment also enhances subjects' donation behavior. For this purpose, we gave the participants the opportunity to donate some or all of their 20 Swiss Franc voucher to an environmental organization. Participants were first asked whether they want to donate, and thereafter received a list of three well-known pro-environmental organizations such as the World Wildlife Fund (WWF), Greenpeace, and Pro Natura. Furthermore, we added an open field in which subjects could name any organization to which they wanted to donate. Overall, $13 \%$ of our participants donated some money to a pro-environmental organization. However, donating was not related to any treatment group or any other variable we took into consideration in any model displayed in Table 7. 
Table 6. The short Crowne Marlowe Social Desirability Scale of Clancy (1971).

\begin{tabular}{clc}
\hline & & Polarity \\
\hline (1) & No matter who I'm talking to, I'm always a good listener. & $\mathrm{T}$ \\
(2) & I sometimes try to get even, rather than forgive and forget. & $\mathrm{F}$ \\
(3) & I have never been irked when people expressed ideas very different from my own. & $\mathrm{T}$ \\
(4) & I never hesitate to go out of my way to help someone in trouble. & $\mathrm{T}$ \\
(5) & I never resent being asked to return a favour. & $\mathrm{T}$ \\
(6) & On occasion I have had doubts about my ability to succeed in life. & $\mathrm{F}$ \\
(7) & There have been times when I felt like rebelling against people in authority even though I knew & $\mathrm{F}$ \\
(8) & Before voting I thoroughly investigate the qualifications of all the candidates. & $\mathrm{T}$ \\
(9) & If I could get into a movie without paying and be sure I was not seen, I would probably do it. & $\mathrm{F}$ \\
(10) & At times I have really insisted on having things my own way. & $\mathrm{F}$ \\
\hline
\end{tabular}

Note: Each item has two answer categories, true (T) and false (F). Respondents receive a score of one for each item answered in the direction of social desirability, for example, answering $\mathrm{T}$ to the first question or answering $\mathrm{F}$ to the second question. $\mathrm{N}=353$, minimum $=2$, maximum $=10$, mean $=7.26$, standard deviation $=1.63$.

Table 7. The results of the logistic regression of charitable giving to a pro-environmental organization on various other variables.

\begin{tabular}{|c|c|c|}
\hline $\begin{array}{c}\text { Model } \\
\text { Dependent Variable }\end{array}$ & \multicolumn{2}{|c|}{ Donation (Yes/No) } \\
\hline Positive video & $\begin{array}{l}-0.07 \\
(0.46)\end{array}$ & $\begin{array}{l}-0.58 \\
(0.53)\end{array}$ \\
\hline Negative video & $\begin{array}{c}0.21 \\
(0.42)\end{array}$ & $\begin{array}{l}-0.06 \\
(0.44)\end{array}$ \\
\hline Mixed video & $\begin{array}{l}-0.10 \\
(0.47)\end{array}$ & $\begin{array}{l}-0.36 \\
(0.51)\end{array}$ \\
\hline Sex: female & & $\begin{array}{c}0.09 \\
(0.45)\end{array}$ \\
\hline Age & & $\begin{array}{l}-0.00 \\
(0.01)\end{array}$ \\
\hline Children (yes/no) & & $\begin{array}{c}-0.78^{+} \\
(0.41)\end{array}$ \\
\hline Equivalence income & & $\begin{array}{c}0.08 \\
(0.05)\end{array}$ \\
\hline Highly educated & & $\begin{array}{l}-0.10 \\
(0.36)\end{array}$ \\
\hline Empathy & & $\begin{array}{c}0.02 \\
(0.03)\end{array}$ \\
\hline Social approval & & $\begin{array}{c}0.04 \\
(0.06)\end{array}$ \\
\hline Importance of money & & $\begin{array}{c}-0.07^{+} \\
(0.04)\end{array}$ \\
\hline Conservative vs. liberal & & $\begin{array}{l}0.12^{+} \\
(0.07)\end{array}$ \\
\hline Left vs. right & & $\begin{array}{l}-0.03 \\
(0.12)\end{array}$ \\
\hline Social desirability & & $\begin{array}{c}0.03 \\
(0.12)\end{array}$ \\
\hline Constant & $\begin{array}{c}-1.97^{* * *} \\
(0.31)\end{array}$ & $\begin{array}{l}-2.24 \\
(2.04)\end{array}$ \\
\hline $\mathrm{n}$ & 360 & 330 \\
\hline Pseudo $\mathrm{R}^{2}$ & 0.00 & 0.06 \\
\hline
\end{tabular}

\section{Discussion}

Our results show that videos about the beauty and endangerment of nature can indeed elevate environmental concern even among members of conservative parties that are traditionally very skeptical about environmental issues. Thus, we confirm former findings that were mainly generated 
with samples of university students. However, only the mixed video-which started with positive messages (beauty of nature) followed by scenes that show the destruction of nature-was effective. The group that saw this video showed concern values that were higher by 0.44 standard deviations. Hence, the effect is visible but modest. However, those who saw the video did not donate more than participants in the control group. This result underlines the notion that environmental behavior cannot be easily altered simply by providing information trying to persuade individuals to act pro-environmentally. Additionally, our experiment only shows that there is a modest short-term effect. Whether, and for how long, it lasts is another question left for further research. Thus, the good news is that pro-environmental videos do have, at least, a positive short-term effect, even among very skeptical individuals. Additionally, the results suggest that such videos do not cause reactivity since none of the treatment groups showed lower values as compared to the control groups. The bad news is, however, that the effect of such videos is limited and that they do not seem to elevate pro-environmental behavior, at least not in form of donations to pro-environmental organizations.

At first sight, one possible argument against our result is that it could have been driven by social desirability or the experimenter demand effect. However, if that were the case then the effect should also appear in the negative and positive video treatment groups. The fact that this is not the case speaks against a demand effect in our experiment. Moreover, we measured social desirability according to the well-known Crowne-Marlowe scale and incorporated it into the model (Figure 3). The results show that the effect is negative. This suggests that in our sample, the socially desirable response is to show less, not more, environmental concern. This reconfirms the notion that we are dealing with a highly environmentally skeptical group for which it is socially desirable to not be concerned with environmental issues.

Finally, one might wonder why only the mixed video consisting of both a positive and a negative video sequence is effective. One possible interpretation is that the mixed video does contain the most information, whereas the other two treatment groups received only information on either positive or negative things. This result also contradicts the interpretation that the videos simply work as a primer by simply activating already-existing information. If priming were the reason, one would expect that the positive or negative video would perform equally well.

Taken together, our results suggest that videos promoting environmental concern do have a small effect which is due to new information they convey to subjects. Hence, educational curricula and environmental organizations are well advised to use videos to convince skeptical subjects. The problem, probably, is how to get skeptical groups to watch such movies.

Author Contributions: Both authors contributed equally to the manuscript. All authors have read and agreed to the published version of the manuscript.

Funding: This research received no external funding

Conflicts of Interest: The authors declare no conflict of interest.

Ethics Statement: Since this study is based on a voluntary survey of the adult population, it does not fall under the Human Research Act according to local (Swiss) law. Hence, it does not require authorization from an ethics committee. 


\section{Appendix A}

Table A1. The results of the linear ordinary least squares regression of environmental concern on various other variables (see also Figure 3).

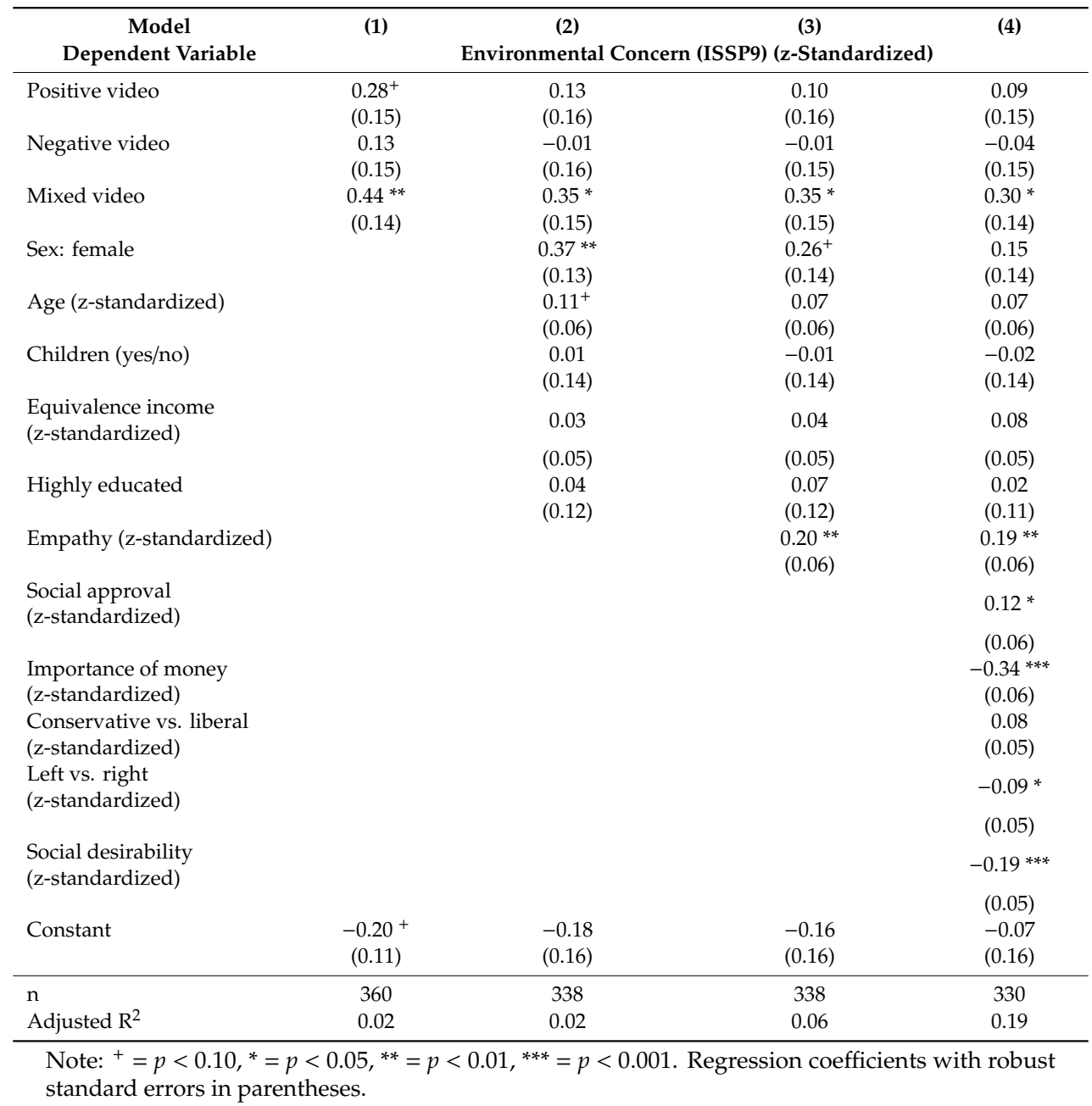

\section{References}

1. McCright, A.M.; Xiao, C.; Dunlap, R.E. Political polarization on support for government spending on environmental protection in the USA, 1974-2012. Soc. Sci. Res. 2014, 48, 251-260. [CrossRef] [PubMed]

2. Clark, A.; Justwan, F.; Carlisle, J.E.; Clark, M. Polarization politics and hopes for a green agenda in the United States. Environ. Politics 2019. [CrossRef]

3. Franzen, A.; Vogl, D. Two decades of measuring environmental attitudes. A comparative analysis of 33 countries. Glob. Environ. Chang. 2013, 23, 1001-1008. [CrossRef]

4. SVP Schweiz. Ich Kämpfe Gegen Den Missbrauch Des Klimawandels Für Politische Zwecke. Available online: https://www.svp.ch/partei/publikationen/extrablatt/extrablatt-juni-2019-vernunft-statt-ideologie/ichkaempfe-gegen-den-missbrauch-des-klimawandels-fuer-politische-zwecke/ (accessed on 7 April 2020).

5. Combes, J.-L.; Hamit-Haggar, M.; Schwartz, S. A multilevel analysis of the determinants of willingness to pay to prevent environmental pollution across countries. Soc. Sci. J. 2018, 55, 284-299. [CrossRef]

6. Post, D.; Meng, Y. Does schooling foster environmental values and action? A cross-national study of priorities and behaviors. Int. J. Educ. Dev. 2018, 60, 10-18. [CrossRef]

7. Blas, M.M.; Alva, I.E.; Carcamo, C.P.; Cabello, R.; Goodreau, S.M.; Kimball, A.M.; Kurth, A.E. Effect of an online video-based intervention to increase HIV testing in men who have sex with men in Peru. PLoS ONE 2010, 5, e10448. [CrossRef] 
8. Roberto, A.J.; Meyer, G.; Johnson, A.J.; Atkin, C.K. Using the extended parallel process model to prevent firearm injury and death: Field experiment results of a video-based intervention. J. Commun. 2000, 50, 157-175. [CrossRef]

9. Stanczyk, N.E.; Smit, E.S.; Schulz, D.N.; de Vries, H.; Bolman, C.; Muris, J.W.M.; Evers, S.M.A.A. An economic evaluation of a video- and text-based computer-tailored intervention for smoking cessation: A cost-effectiveness and cost-utility analysis of a randomized controlled trial. PLoS ONE 2014, 9, e110117. [CrossRef]

10. Thornton, J.D.; Patrick, B.; Sullivan, C.; Albert, J.M.; Wong, K.A.; Allen, M.D.; Kimble, L.; Mekesa, H.; Bowen, G.; Sehgal, A.R. Comparing web-based video interventions to enhance university student willingness to donate organs: A randomized controlled trial. Clin. Transplant. 2019, 33, e13506. [CrossRef]

11. Vogt, S.; Zaid, N.A.M.; Ahmed, H.E.F.; Fehr, E.; Efferson, C. Changing cultural attitudes towards female genital cutting. Nature 2016, 538, 506-509. [CrossRef]

12. Yousuf, H.; Narula, J.; Zwetsloot, P.-P.; Hofstra, M.; de Levita, A.; Scherder, E.; van Rossum, B.; Hofstra, L. Using entertainment to improve lifestyles and health. Lancet 2019, 394, 119-120. [CrossRef]

13. Johnson, D.; Wilson, D.B.; Maguire, E.R.; Lowrey-Kinberg, B.V. Race and perceptions of police: Experimental results on the impact of procedural (in)justice. Justice Q. 2017, 34, 1184-1212. [CrossRef]

14. Maguire, E.R.; Lowrey, B.V.; Johnson, D. Evaluating the relative impact of positive and negative encounters with police: A randomized experiment. J. Exp. Criminol. 2017, 13, 367-391. [CrossRef]

15. Skurka, C.; Quick, B.L.; Reynolds-Tylus, T.; Short, T.; Bryan, A.L. An evaluation of a college campus emergency preparedness intervention. J. Saf. Res. 2018, 65, 67-72. [CrossRef] [PubMed]

16. Moss-Racusin, C.A.; Pietri, E.S.; Hennes, E.P.; Dovidio, J.F.; Brescoll, V.L.; Roussos, G.; Handelsman, J. Reducing STEM gender bias with VIDS (Video Interventions for Diversity in STEM). J. Exp. Psychol. Appl. 2018, 24, 236-260. [CrossRef]

17. Zelenski, J.M.; Dopko, R.L.; Capaldi, C.A. Cooperation is in our nature: Nature exposure may promote cooperative and environmentally sustainable behavior. J. Environ. Psychol. 2015, 42, 24-31. [CrossRef]

18. Janpol, H.L.; Dilts, R. Does viewing documentary films affect environmental perceptions and behaviors? Appl. Environ. Educ. Commun. 2016, 15, 90-98. [CrossRef]

19. Shreedhar, G.; Mourato, S. Experimental evidence on the impact of biodiversity conservation videos on charitable donations. Ecol. Econ. 2019, 158, 180-193. [CrossRef]

20. Jacobson, S.K.; Morales, N.A.; Chen, B.; Soodeen, R.; Moulton, M.P.; Jain, E. Love or Loss: Effective message framing to promote environmental conservation. Appl. Environ. Educ. Commun. 2019, 18, 252-265. [CrossRef]

21. Sunstein, C.R.; Reisch, L.A.; Kaiser, M. Trusting nudges? Lessons from an international survey. J. Eur. Public Policy 2019, 26, 1417-1443. [CrossRef]

22. Thaler, R.; Sunstein, C.R. Nudge: Improving Decision about Health, Wealth, and Happiness; Yale University Press: New Haven, CT, USA, 2008.

23. Franzen, A.; Mader, S. Testing the Measurement of Environmental Concern: Do Single Items Outperform Multi-Item Scales? University of Bern: Bern, Switzerland, 2020.

24. Holm, S. A simple sequentially rejective multiple test procedure. Scand. J. Stat. 1979, 6, 65-70.

25. Davis, M.H. Measuring individual differences in empathy: Evidence for a multidimensional approach. J. Personal. Soc. Psychol. 1983, 44, 113-126. [CrossRef]

26. Franzen, A.; Mader, S.; Winter, F. Contagious yawning, empathy, and their relation to pro-social behavior. J. Exp. Psychol. Gen. 2018, 147, 1950-1958. [CrossRef] [PubMed]

27. Crowne, D.P.; Marlowe, D. A new scale of social desirability independent of psychopathology. J. Consult. Psychol. 1960, 24, 349-354. [CrossRef] [PubMed]

28. Clancy, K. Systematic Bias in Field Studies of Mental Illness. Ph.D. Thesis, New York University, New York, NY, USA, 1971.

29. Franzen, A.; Mader, S. Do phantom questions measure social desirability? Methods Data Anal. 2019, 13, 37-57. 